\title{
1.94 GHz CW Modelocked Ytterbium-Doped Bismuthate Glass Waveguide Laser
}

\author{
Adam Lancaster, ${ }^{1 *}$ Amol Choudhary, ${ }^{2,3}$ Nitin S. Jha, ${ }^{1}$ Rose Mary, ${ }^{1}$ David P. Shepherd ${ }^{2}$ and Ajoy. K. Kar ${ }^{1}$ \\ ${ }^{1}$ Institute of Photonics and Quantum Sciences, Heriot-Watt University, Edinburgh, EH14 4AS, UK \\ ${ }^{2}$ Optoelectronics Research Centre, University of Southampton, Highfield, Southampton, SO17 1BJ, UK \\ ${ }^{3}$ Current Address: CUDOS, School of Physics, University of Sydney, NSW, Australia, 2006 \\ al164@hw.ac.uk
}

\begin{abstract}
A $1.94 \mathrm{GHz} \mathrm{CW}$ modelocked ytterbium-doped bismuthate waveguide laser is presented. The waveguide was fabricated using the ultrafast laser inscription technique. Pulse energy of 30.9 $\mathrm{pJ}$ and pulse duration of $1.1 \mathrm{ps}$ are inferred from the spectral width.

OCIS codes: (130.2755) Glass waveguides; (140.3390) Laser materials processing; (140.3615) Lasers, ytterbium; (140.7090) Ultrafast lasers
\end{abstract}

\section{Introduction}

In recent years there has been much interest in the development of ultra-fast laser systems with pulse repetition frequency (PRF) greater than $1 \mathrm{GHz}$. This has been motivated by many applications in non-linear microscopy, frequency metrology and optical communications [1-3]. Due to engineering difficulties it can be challenging to achieve a laser with a fundamental PRF greater than $1 \mathrm{GHz}$ with a standard bulk cavity solid-state laser system because the fundamental PRF is directly linked to the optical cavity length [4]. In order to achieve a short optical cavity length we propose using a waveguide laser, as this allows the cavity mirrors to be placed at the end facets of the waveguide thus reducing the cavity length to that of the gain medium. R. Mary et al. [5] reported a highly efficient monolithic waveguide laser in $\mathrm{Yb}$-doped bismuthate glass $(\mathrm{BG})$ operating in continuous wave $(\mathrm{CW})$ mode. The slope efficiency of the laser was $79 \%$, which is close to the quantum defect limit. Later work by R. Mary demonstrated a Q-switched modelocked laser with $\mathrm{Yb}: \mathrm{BG}$ waveguides using a graphene saturable output coupler with a PRF of $1.5 \mathrm{GHz}$ [6]. Lagatsky et al. [7] reported that group velocity dispersion (GVD) can be controlled by accurately changing the length of the gap between the end facet of the waveguide and one of the cavity mirrors. This method of the GVD control was used to demonstrate a fundamentally mode-locked femtosecond laser in Yb:IOG-1 with a PRF of $15.2 \mathrm{GHz}$ and pulse energy of $1.8 \mathrm{pJ}$ [7]. By combining the work of R. Mary and A. A. Lagatsky we aim to demonstrate a compact, highly efficient $\mathrm{CW}$ modelocked in the near infrared with a fundamental PRF of $>1 \mathrm{GHz}$.

In this letter we demonstrated the first $\mathrm{CW}$ modelocked ytterbium-doped bismuthate waveguide laser with a PRF of $1.94 \mathrm{GHz}$. A SESAM was used to passively modelocked the laser. The waveguides where fabricated using ultrafast laser inscription.

\section{Waveguide fabrication}

The sample of Yb-doped bismuthate glass (Yb:BG) used in this investigation had a $\mathrm{Yb}$ dopant concentration of $1.6 \times$ $10^{26} \mathrm{~m}^{-3}$, refractive index of 2.03 at $1304 \mathrm{~nm}$ and dimensions of $40 \mathrm{~mm} \times 15 \mathrm{~mm} \times 2 \mathrm{~mm}$. The waveguides were fabricated in the sample at a depth of $200 \mu \mathrm{m}$ using ultrafast laser inscription (ULI). ULI utilizes the nonlinear absorption of a femtosecond pulse tightly focused below the surface of a transparent dielectric material. The high irradiance at the focus allows nonlinear processes such as multi-photon, tunneling, and avalanche ionization to transfer energy to the material lattice [8]. This energy transfer can result in a change in refractive index at the focus of the laser beam. In the case of $\mathrm{Yb}: \mathrm{BG}$ an increase in refractive index is utilized for the waveguide fabrication. In order to achieve a symmetrical mode field profile the multi-scan technique is used [9]. This process translates the sample through the focus of the beam 20 times with a small $(0.4 \mu \mathrm{m})$ lateral offset in order to achieve a square waveguide cross-section. The inscription laser used in this work was a femtosecond $\mathrm{Yb}$ :fiber laser (IMRA $\mu \mathrm{J} 400$ ) operated at a PRF of $500 \mathrm{kHz}$ with a pulse width of $350 \mathrm{fs}$. The femtosecond laser beam was focused into the sample using a slightly overfilled lens that had a NA of 0.4. A range of inscription parameters where investigated such as laser pulse energy from 40 to 192 $\mathrm{nJ}$ and sample translation speeds from 2 to $12 \mathrm{~mm} / \mathrm{s}$. Full details of the waveguide fabrication and $\mathrm{CW}$ laser characterization are given by R. Mary et al. [5]. 


\section{Laser characterization}

The pump source for this experiment consisted of two PM fiber-coupled 975-nm laser diodes that were combined using polarization multiplexing. An 11-mm-focal-length AR-coated lens was used to couple the pump light into the end facet of the $\mathrm{Yb}: \mathrm{BG}$ waveguide. This lens also collimated the laser output. A $98 \%$ reflective mirror (at the laser wavelength) was used as the output coupler and was butt-coupled to the $\mathrm{Yb}: \mathrm{BG}$ waveguide input facet with a fluorinated liquid (Fluorinert FC-70). The SESAM (Batop GmbH) used in this work had an initial reflection of 99.3 $\%$ at $1050 \mathrm{~nm}$, a modulation depth of $0.4 \%$, saturation fluence of $90 \mu \mathrm{J} / \mathrm{cm}^{2}$ and a relaxation time of $0.5 \mathrm{ps}$. The SESAM was mounted on a translation stage with manual adjustment and a piezo-electric stage to allow fine control of the gap $\Delta \mathrm{x}$. A schematic of the laser cavity design is given in Figure 1.

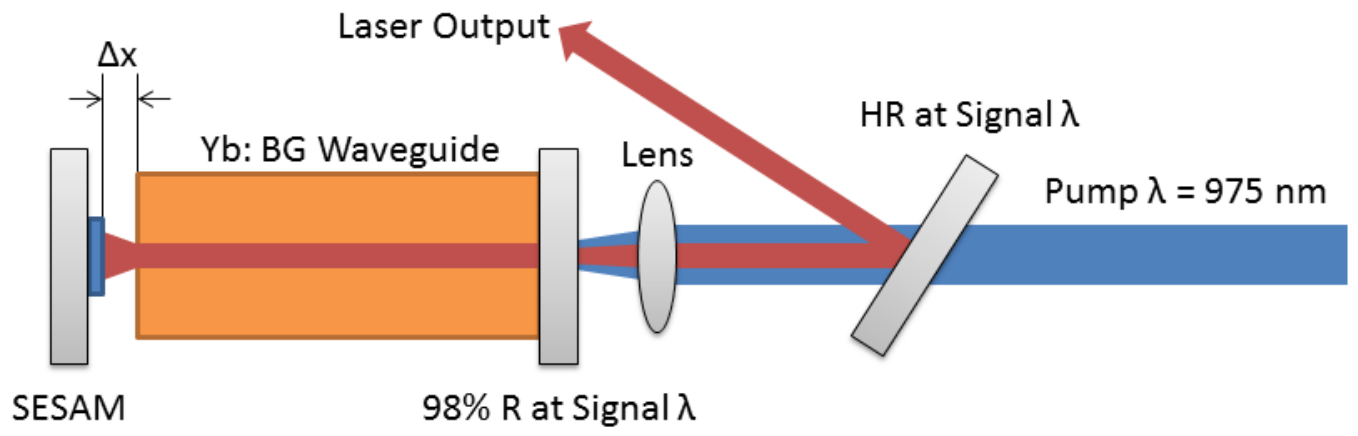

Figure 1: Schematic of laser cavity design used in the demonstration of CW modelocking in $\mathrm{Yb}: \mathrm{BG}$

On initial alignment, the system demonstrated Q-switched modelocking but with very fine adjustment of $\Delta \mathrm{x}$ with the piezoelectric motion controller $\mathrm{CW}$ modelocking was observed with a pump power of $800 \mathrm{~mW}$. The authors confirmed CW modelocking by the RF spectrum shown in Figure 2. From the RF spectrum, the laser was found to have a PRF of $1.94 \mathrm{GHz}$, which is in good agreement with that expected from the cavity length. The average output power of the laser was measured to be $60 \mathrm{~mW}$ with an incident pump power of $800 \mathrm{~mW}$.

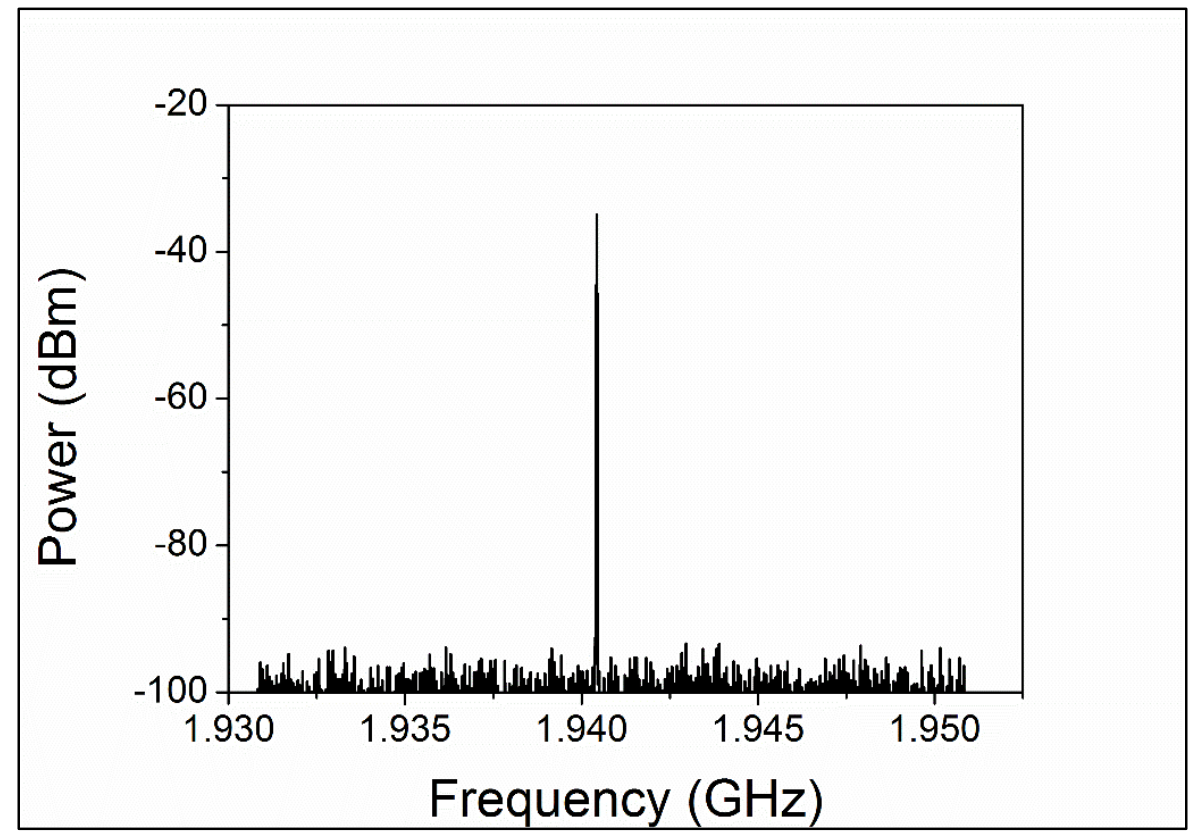

Figure 2: Frequency spectrum of the $\mathrm{Yb}: \mathrm{BG}$ waveguide laser in $\mathrm{CW}$ modelocked operation with $800 \mathrm{~mW}$ of pump light incident on the end facet of the waveguide.

The wavelength spectrum of the laser was measured using an OSA with a resolution of $0.1 \mathrm{~nm}$. The spectrum of the laser during CW modelocked operation shown in Figure 3. The laser emission was centered at $1029 \mathrm{~nm}$ with a 
FWHM of $1 \mathrm{~nm}$. From the FWHM of $1 \mathrm{~nm}$ we can place a lower limit on the pulse duration of $1.1 \mathrm{ps}$, assuming a sech $^{2}$ transform-limited pulse.

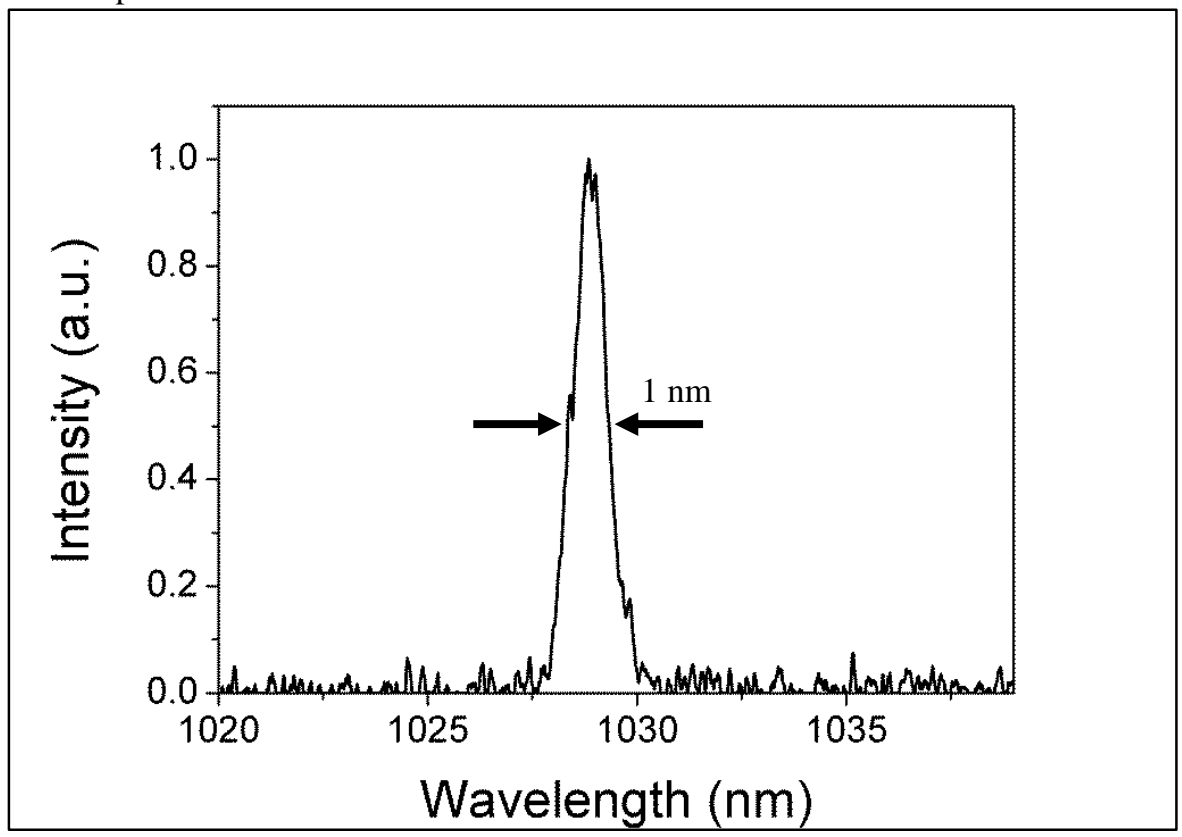

Figure 3: Optical spectra of the CW modelocked Yb:BG waveguide laser operating under $800 \mathrm{~mW}$ of pump excitation. The spectra was centered at $1029 \mathrm{~nm}$ with a FWHM of $1 \mathrm{~nm}$.

\section{Conclusions}

A CW modelocked ULI inscribed waveguide laser has been demonstrated. GHz fundamental PRF CW modelocking was achieved by using an inter-cavity gap for group dispersion compensation. The laser operated at a PRF of 1.94 $\mathrm{GHz}$ with maximum pulse energy of $30.9 \mathrm{pJ}$ at $1029 \mathrm{~nm}$.

\section{References}

[1] S.W. Chu, T.M. Liu, C.K. Sun, C.Y. Lin, and H.J. Tsai, "Real-time second-harmonic-generation microscopy based on a 2-GHz repetition rate Ti:sapphire laser," Opt. Express 11, 933-938 (2003).

[2] Y. J. Chai, C. G. Leburn, A. A. Lagatsky, C. T. A. Brown, R. V. Penty, I. H. White, and W. Sibbett, "1.36-Tb/s Spectral Slicing Source Based on a $\mathrm{Cr}^{4+}$ YAG Femtosecond Laser," J. Lightwave technol. 23, 1319 (2005).

[3] D. J. Jones, S. A. Diddams, J. K. Ranka, A. Stentz, R. S. Windeler, J. L. Hall, and S. T. Cundiff, "Carrier-Envelope Phase Control of Femtosecond Mode-Locked Lasers and Direct Optical Frequency Synthesis," Science 288, 635-639 (2000).

[4] U. Keller, "Recent developments in compact ultrafast lasers," Nature 424, 831-838 (2003).

[5] R. Mary, S. J. Beecher, G. Brown, R. R. Thomson, D. Jaque, S. Ohara, and A. K. Kar, "Compact, highly efficient ytterbium doped bismuthate glass waveguide laser," Opt. Lett. 37, 1691-1693 (2012).

[6] R. Mary, G. Brown, S. J. Beecher, F. Torrisi, S. Milana, D. Popa, T. Hasan, Z. Sun, E. Lidorikis, S. Ohara, A. C. Ferrari, and A. K. Kar, "1.5 GHz picosecond pulse generation from a monolithic waveguide laser with a graphene-film saturable output coupler," Opt. Express 21, 7943-7950 (2013).

[7] A. A. Lagatsky, A. Choudhary, P. Kannan, D. P. Shepherd, W. Sibbett, and C. T. A. Brown, "Fundamentally mode-locked, femtosecond waveguide oscillators with multi-gigahertz repetition frequencies up to $15 \mathrm{GHz}$," Opt. Express 21, 19608-19614 (2013).

[8] R. R. Gattass, and E. Mazur, "Femtosecond laser micromachining in transparent materials," Nat. Photonics 2, 219-225 (2008).

[9] H. T. Bookey, R. R. Thomson, N. D. Psaila, A. K. Kar, N. Chiodo, R. Osellame, and G. Cerullo, "Femtosecond Laser Inscription of Low Insertion Loss Waveguides in Z-Cut Lithium Niobate," IEEE Photonic. Tech. L. 19, 892-894 (2007). 